\title{
Statistical Modeling of the Influence of Electron Degeneracy on the Interatomic Interactions
}

\author{
Anatoly M. Dolgonosov \\ Vernadsky Institute of Geochemistry and Analytical Chemistry, Russian Academy of Sciences, Moscow, Russia \\ E-mail: amdolgo@mail.ru
}

Received April 15, 2010; revised July 2, 2010; accepted July 30, 2010

\begin{abstract}
It is shown that electrons forming simple and multiple covalent bonds may have different contributions to the interatomic interactions due to the degeneracy of electron states. A simple relationship between the length of covalent bond, its order and atomic numbers of the interacting atoms is deduced.
\end{abstract}

Keywords: Interatomic Interactions, Covalent Bond, Degeneracy of Electron States, Theory of Generalized Charges, Bond Length Ratio

\section{Introduction}

In the semiempirical methods describing interatomic interactions, the contribution to the interaction energy of $\pi$-bond is assumed to be larger than that of $\sigma$-bond $[1,2]$. The ratio of $\pi$ - to $\sigma$-electron weight factors equal to 1.41 was empirically determined in $[3,4]$ and checked many times for the adsorption of unsaturated hydrocarbons. We will show that this ratio can be evaluated from the characteristics of covalent bonds (and vice versa).

It follows from the theoretical and empirical equations found by London, Heitler, Lennard-Jones, and others that the parameters of atoms symmetrically enter the expression for interatomic bond energy [5]. A quasi-classical method for describing the self-consistent field of multicomponent electron gas was developed in [6]. This method enables us to express the interatomic interaction energy. In particular it is shown that this energy is associated with the volume $V$ of each atom through a symmetrical operation - volumes product

$$
\begin{aligned}
& E_{12}=f\left(X_{12}, r_{12}\right) ; \\
& X_{12}=V_{1} V_{2}=\sum_{i(1)} v_{i} \sum_{j(2)} v_{j}
\end{aligned}
$$

where $r_{12}$ is the internuclear distance; $v$ is a "volume" of one electron, or the elementary volume, which is equal to unity for a nondegenerate electron; $V$ is the electronic volume of the atom equal to the sum of elementary volumes of its electrons; the indices enumerate all electrons participating in bonding; and the figures at the summation symbol indicate summation over every electron of the corresponding atom.

According to [6], the shielding radius, which is inversely proportional to the square root of the height of the potential barrier that the electron overcomes, can be used as a criterion of the participation of the electron in interatomic interactions. A sphere centered at the atomic nucleus with radius equal to the shielding radius of electrons (the shielding sphere) bounds the electrons that participate in bonding. An electron contributes to the electronic volume of its atom only if the nucleus of the atom with which the interaction is considered is situated within the electron shielding sphere.

It is very important to distinguish between the electronic volume, the key concept of the theory of generalized charges developed in [6], and the corresponding number of electrons, though these characteristics often quantitatively coincide. It will be shown below that the elementary volume of a degenerate electron larger than that of a nondegenerate one, i.e. larger than unity.

\section{Theory}

Let us consider the sums in (1) in detail

$$
\begin{aligned}
& V_{1} V_{2}=\sum_{i(1)} v_{i} \sum_{j(2)} v_{j}= \\
& =\frac{1}{2}\left(\sum_{i j} v_{i} v_{j}-\sum_{i j(1)} v_{i} v_{j}-\sum_{i j(2)} v_{i} v_{j}\right)
\end{aligned}
$$

where $v_{i}=v_{j}=1$. The summation is over all possible pairs of electrons. The coefficient $1 / 2$ appears because there are no limitations on the permutation of indices.

Let us apply (2) to pairs of $\pi$ - and $\sigma$-bond electrons. For a $\sigma$-bond, the orbital moment projection $(m)$ of its electrons onto the internuclear axis has a single (zero) value. A pair of $\sigma$-electrons has therefore one state only

$$
v_{\sigma}^{2} \equiv \frac{1}{2}\left(v_{i(1)} v_{j(2)}+v_{j(1)} v_{i(2)}\right)=1 .
$$


For $\pi$-bond electrons, the orbital moment projections onto the same axis take equal values, either +1 or -1 (depending on whether the right- or left-handed coordinate system is used; here we use atomic units of moment). We therefore have four terms for $\pi$-bond electrons

$$
\begin{aligned}
& v_{\pi}^{2} \equiv \frac{1}{2}\left[\left.v_{i(1)} v_{j(2)}\right|_{m=+1}+\left.v_{j(1)} v_{i(2)}\right|_{m=+1}+\left.v_{i(1)} v_{j(2)}\right|_{m=-1}+\right. \\
& \left.\left.v_{j(1)} v_{i(2)}\right|_{m=-1}\right]=2
\end{aligned}
$$

This gives $v_{\pi}=\sqrt{2}$. It follows that the $n$-fold state of degeneracy corresponds to the $n$-fold increase in the sum of pair electronic products, that enhances the elementary volume of the pair of bond electrons, $v_{e}=\sqrt{n}$. It is necessary to bear in mind that the electron balance condition imposes the following restriction: if $\sum V_{a}=\sum Z_{a}$, then $v_{e} \equiv 1$ and, vice versa, if at least one $v_{e}$ value is larger than 1 , then $\sum V_{a}<\sum Z_{a}$.

The role of the product of volumes in describing the interaction of atoms is clarified by the following identity:

$$
\sqrt{V_{1} V_{2}}=\frac{1}{2}\left[\left(\sqrt{V_{1}}+\sqrt{V_{2}}\right)^{2}-V_{1}-V_{2}\right]
$$

The appropriately normalized electronic volume of an atom corresponds to the probability of that its electrons belong to the bond under consideration. In terms of probability, the expression in brackets is the excess value, which appears as a result of bond formation.

On the other hand, the covalent bond energy is a function of the excess electron density in the internuclear space

$$
\begin{aligned}
& \bar{\rho}\left(r_{12}\right) \propto\left(Z_{1}+Z_{2}\right)\left[\int_{\left(l=r_{12}\right)} \Psi * \Psi d \tau-\int_{(l=\infty)} \Psi * \Psi d \tau\right]= \\
& =\left(Z_{1}+Z_{2}\right)\left[\overline{\psi^{*}}\left(r_{12}\right) \bar{\psi}\left(r_{12}\right)-A\right], \\
& A=\overline{\psi^{*}}(\infty) \bar{\psi}(\infty)=\frac{1}{2}
\end{aligned}
$$

where $Z_{1}, Z_{2}$ are the charges of nuclei in the elementary charge units; $d \tau$ is the space volume element; $l$ is the interatomic distance; and the bars denote the averaging over the scale indicated in parentheses, which coincides with one of the arguments of the functional relation. The first integral equals the probability for an atomic electron to occur between the planes passing through the nuclei normally to the interatomic axis at distance $r_{12}$ from each other. The second integral gives the same probability at infinite interatomic distance. The integration in (4) is performed taking into account that the electronic wave function in the internuclear space depends on bond length. When atoms are infinitely separated, the excess electron density is zero, and exactly one-half of all the electrons occur in the internuclear space.

We can therefore write $E_{12}=F\left(\bar{\rho}\left(r_{12}\right)\right)=f\left(X_{12}, r_{12}\right)$ that gives

$$
\bar{\rho}\left(r_{12}\right)=g\left(V_{1} V_{2}\right)
$$

Comparing (3) and (4) by their sense and taking into account (5), we obtain

$$
\sqrt{V_{1} V_{2}}=\left(Z_{1}+Z_{2}\right)\left(\psi\left(r_{12}\right) \psi^{*}\left(r_{12}\right)-\frac{1}{2}\right) \propto \bar{\rho}\left(r_{12}\right)
$$

According to (6), the excess density in the internuclear space is proportional to the geometric mean of the probabilities for atomic electrons to take part in the bond under consideration.

To simplify (6), let us make the substitution

$$
\bar{\psi}\left(r_{12}\right)=\frac{1}{\sqrt{2}} \frac{\sqrt{Z_{1}}}{\sqrt{Z_{1}+Z_{2}}} \overline{\psi_{1}}\left(r_{12}\right)+\frac{1}{\sqrt{2}} \frac{\sqrt{Z_{2}}}{\sqrt{Z_{1}+Z_{2}}} \overline{\psi_{2}}\left(r_{12}\right)
$$

where $\overline{\psi_{a}}\left(r_{12}\right) \equiv \psi_{a}=\exp \left(i \varphi_{a}\right) ; a=1,2$. We obtain:

$$
\frac{1}{2}\left(\psi_{1}^{*} \psi_{2}+\psi_{1} \psi_{2}^{*}\right) \equiv \cos \left(\varphi_{1}-\varphi_{2}\right)=\sqrt{\frac{V_{1} V_{2}}{Z_{1} Z_{2}}} \equiv \alpha
$$

Let us clear the combinatorial meaning of $\alpha$ : its square is the relative part of the cases when a pair of the particles belonging to two nonoverlapping sets of $Z_{1}$ and $Z_{2}$ particles occurs among the set of $V_{1}+V_{2}$ particles, where $V_{1}$ particles belong to the first set and $V_{2}$ particles, to the second one. This interpretation suffers from the shortcoming that the electronic volume is a more complex concept that the number of electrons.

The above-mentioned quantity $\overline{\psi_{a}}\left(r_{12}\right) \equiv \psi_{a}$ is the characteristic of atom depending on bond length. In such a case the $\varphi_{a}$ phase is a function of the scalar product of the wave vector of atomic electrons $\left(\mathbf{k}_{a}\right)$ and the internuclear vector

$$
\begin{aligned}
& \varphi_{a}=\varphi\left(\mathbf{k}_{a} \mathbf{r}_{a a^{\prime}}\right)=\sum_{i} A_{i}\left(\mathbf{k}_{a} \mathbf{r}_{a a^{\prime}}\right)^{i} ; \\
& a, a^{\prime}=1,2 ; a^{\prime} \neq a ; i=0, \pm 1, \pm 2, \ldots
\end{aligned}
$$

The presence of even exponents in expansion (9) is inessential because of their zero contribution to the difference of phases in (8) when the atoms are identical. For the same reason, at least one of the odd constants $A_{i}$ in (9) is nonzero. When the internuclear distance in its tending to zero falls beneath a certain value, the number of electrons forming the bond becomes nonzero. Negative exponents are therefore absent in (9). The absolute term $(i$ $=0)$ in (8) is annulled and therefore does not play any role. Thus, we can keep in (9) only the linear term, and set, without loss of generality, the constant $A_{1}$ equal to 
unity

$$
\varphi_{a}=\left(\mathbf{k}_{a} \mathbf{r}_{a a^{\prime}}\right) ; \varphi_{1}-\varphi_{2}=\left(\mathbf{r}_{12}\left(\mathbf{k}_{1}+\mathbf{k}_{2}\right)\right)
$$

Rewrite expression (8) taking into account (10):

$$
\alpha=\cos \left(\mathbf{r}_{12}\left(\mathbf{k}_{1}+\mathbf{k}_{2}\right)\right)=\cos \left(r_{12} \sum k_{r}\right),
$$

where $\sum k_{r}$ is the sum of projections of the wave vectors of bond electrons on the bond axis. Finding this sum from (11), we get

$$
\sum k_{r}= \pm r_{12}^{-1} \arccos \alpha
$$

The remarkable feature of $\sum k_{r}$ is its independence of $\pi$-electrons of the bond, because the limit value of $\pi$-electron moment projection on the bond axis corresponds to the zero projection of its wave vector in this direction

$$
\sum k_{r}=k_{\sigma 1 r}+k_{\sigma 2 r}, \sum k_{\pi r} \equiv 0
$$

Combining (11) with (12) and (13), we find the general expression for a covalent bond

$$
\alpha^{\prime}=\cos \left(r_{12}^{\prime} \sum k_{r}\right)=\cos \left(\frac{r_{12}^{\prime}}{r_{12}} \arccos \alpha\right)
$$

where primed and unprimed values relate to different cases of the bond between the same atoms. Note that (14) is valid for both double and triple bonds. Similar to (8), we can write

$$
\alpha^{\prime}=\sqrt{\frac{V_{1}^{\prime} V_{2}^{\prime}}{Z_{1} Z_{2}}},
$$

where $V^{\prime}$ differs from the single bond property $V$ by the replacement of one or two electrons by $\pi$-electrons of double or triple bond, correspondingly. Thus, the number of electrons forming a covalent bond is independent of its order and, due to the above-introduced normalization of electronic volume, is equal to the electronic volume of atom for the case of single bond $\left(V_{a}\right)$. Taking into account the value $v_{\pi}=\sqrt{2}$ obtained above, we find

$$
V_{a}^{\prime}-V_{a}=(\sqrt{2}-1)(n-1)
$$

where $n=1,2,3$ is the bond order. In particular, it follows from (14) that for homo-nuclear bonds

$$
V^{\prime}=Z \cos \left(\frac{r_{12}^{\prime}}{r_{12}} \arccos \frac{V}{Z}\right)
$$

It is quite natural to assume that $V_{a}$ can differ from $Z_{a}$ by the number of closed shell electrons (for which the shielding radius is smaller than that for outer shell electrons), equal to two for the second-period atoms that yields $V_{a}=Z_{a}-2$. This expression in combination with (15) and (16) gives after simple transformations (see
Appendix)

$$
\begin{aligned}
\frac{r_{12}^{\prime}}{r_{12}} & =\arcsin \sqrt{\frac{2-(\sqrt{2}-1)(n-1)}{2 Z}} / \arcsin \sqrt{\frac{1}{Z}} \approx \\
& \approx \sqrt{1-\frac{(\sqrt{2}-1)(n-1)}{2}}
\end{aligned}
$$

The last result valid for large atomic numbers is valuable due to its independence of the kind of atoms, displaying only the dependence of the bond order.

The calculation using (18) gives $\frac{r_{12}^{\prime}}{r_{12}}=0.890$ for double bond and $\frac{r_{12}^{\prime \prime}}{r_{12}}=0.765$ for triple bond. Formula (18) is approximately valid for hetero-nuclear compounds as well.

The comparison of the theoretical result obtained with experimental data is given in the Table.

For some possible compounds there is no information on bond length. This lack of knowledge can be eliminated by theoretical forecast. For example, the triple bond of boron with carbon or nitrogen is possible in principle. Its length will be about $24 \%$ shorter than the corresponding single bond.

\section{Conclusions}

Thus, there is good compliance between experimental and theoretical values that confirms the necessity to distinguish in interatomic interactions the contributions of differently degenerated electrons. The contribution of $\pi$-electron to such an additive property of the interactive atom as its electronic volume is in $\sqrt{2}$ times larger than that of $\sigma$-electron. This effect can be explained by different symmetries of the states with different degeneracy of $\sigma$ - and $\pi$-electrons. In present work, the simple expression $(17,18)$ for covalent bond length ratio which shows strong influence of bond orders and weak dependence on atomic numbers is obtained.

Table. Ratios of the bond lengths for compounds of the second-period elements: reference data $[7,8]$ versus calculation results.

\begin{tabular}{lll}
\hline Atoms bonded & $\begin{array}{l}\text { Bond length ratio: } \\
\text { double to single }\end{array}$ & $\begin{array}{l}\text { Bond length ratio: } \\
\text { triple to single }\end{array}$ \\
\hline $\mathrm{C}-\mathrm{C}$ & 0.865 & 0.778 \\
$\mathrm{~N}-\mathrm{N}$ & 0.862 & 0.757 \\
$\mathrm{O}-\mathrm{O}$ & $0.813\left(\mathrm{O}_{2}\right) ;$ & $0.766\left(\mathrm{O}_{2}^{+}\right)[8]$ \\
$\mathrm{C}-\mathrm{N}$ & $0.861\left(\mathrm{O}_{3}\right)$ & 0.786 \\
$\mathrm{C}-\mathrm{O}$ & 0.910 & 0.791 \\
$\mathrm{~N}-\mathrm{O}$ & 0.852 & $0.779\left(\mathrm{NO}^{+}\right)[8]$ \\
$\begin{array}{l}\text { Theory: } \\
\text { formula (18) }\end{array}$ & 0.897 & 0.765 \\
\hline
\end{tabular}




\section{References}

[1] E. V. Kalashnikova, A. V. Kiselev, D. P. Poshkus and K. D. Shcherbakova, "Retention Indices in Gas-Solid Chromatography,” Journal of Chromatography A, Vol. 119, No. 41, 1976, pp. 233-242.

[2] J. A. Pople and D. L. Beveridge, “Approximate Molecular Orbital Theory,” McGraw-Hill, New York, 1970.

[3] A. M. Dolgonosov, "Energy and Molecular Area of the Adsorbate on a Uniform Adsorbent," Doklady Physical Chemistry, Vol. 358, No. 1-3, 1998, pp. 26-30.

[4] A. M. Dolgonosov, "Calculation of Adsorption Energy and Henry Law Constant for Nonpolar Molecules on a

\section{Appendix}

Deduction of (17).

Let us denote

$$
s_{1}=\frac{2-(\sqrt{2}-1)(n-1)}{2 Z}, s_{2}=\frac{1}{Z} .
$$

It is necessary to transform the expression

$$
x=\frac{\arccos \left(1-2 s_{1}\right)}{\arccos \left(1-2 s_{2}\right)} .
$$

Using the formula $\arccos t=\arcsin \sqrt{1-t^{2}}$ yields
Nonpolar Uniform Adsorbent," The Journal of Physical Chemistry B, Vol. 102, No. 24, 1998, pp. 4715-4730.

[5] S. Lundquist and N. H. March (Eds.), "Theory of the Inhomogeneous Electron Gas,” Plenum Press, New York -London, 1983.

[6] A. M. Dolgonosov, "Electron Gas Model and Theory of Generalized Charges for Description of Interatomic Forces and Adsorption,” Librokom/Urss, Moscow, 2009.

[7] A. J. Gordon and R. A. Ford, "The Chemist Companion," Wiley, New York, 1972.

[8] V. A. Rabinovich and Z. Ya. Havin, "Brief Chemical Handbook,” Khimia, St-Petersburg, 1994

$$
x=\frac{\arcsin \sqrt{2 s_{1}\left(2-2 s_{1}\right)}}{\arcsin \sqrt{2 s_{2}\left(2-2 s_{2}\right)}}=\frac{\arcsin 2 \sqrt{s_{1}\left(1-s_{1}\right)}}{\arcsin 2 \sqrt{s_{2}\left(1-s_{2}\right)}} .
$$

Substitution of $s_{i}=\sin ^{2} \varphi_{i}$ leads to

$$
\begin{aligned}
& x=\frac{\arcsin 2 \sqrt{\sin ^{2} \phi_{1} \cos ^{2} \phi_{1}}}{\arcsin 2 \sqrt{\sin ^{2} \phi_{2} \cos ^{2} \phi_{2}}}=\frac{\arcsin \left(\sin 2 \phi_{1}\right)}{\arcsin \left(\sin 2 \phi_{2}\right)}= \\
& =\frac{\phi_{1}}{\phi_{2}}=\frac{\arcsin \sqrt{s_{1}}}{\arcsin \sqrt{s_{2}}}
\end{aligned}
$$

The inverse substitutions of the expressions for $s_{1}, s_{2}$ give formula (17). 